\title{
UTILIZATION OF IR LASER PUMPED ANTI-STOKES EMISSION OF Er-Yb DOPED SYSTEMS FOR IDENTIFICATION OF SECURITIES
}

\author{
A.N. Kuzmin, G.I. Ryabtsev, G.A. Ketko \\ Institute of Pliysics, Belarussian Academy of Sciences, Minsk, Belarus \\ A.Ya. Gorelenko, A.A. Demidovici \\ Science Center "Gosznak" of Belarus, Minsk, Belarus \\ W. Stręk, K. Maruszewicz and P. Dereń \\ Institute for Low Temperature and Structure Research, Polish Academy of Sciences \\ Okólna 2, 50-950 Wrocław, Poland
}

In this paper we present a utilization of anti-Stokes luminescence of Er-Yb systems for identifications of securities. A simple method of detection of an up-conversion phenomenon in such system by means of IR laser operating in the region $960-1010 \mathrm{~nm}$ is proposed.

PACS numbers: 78.55.IIx

\section{Introduction}

The optical properties of rare earth ions may be utilized for identification and protection of securities. The most favourable feature of rare earth elements is a selective absorption or reflection of exciting radiation [1]. For manufacturing of security papers the thin absorbing and/or reflecting layers containing rare earth elements may be used. This imposes on light sources, used for visualization of means of protection, stringent requirements on power, spectral and temporal characteristics.

The cooperative interaction between rare earth ions in pairs or aggregates leads to occurrence of multiphoton process such as two-photon absorption or emission. The phenomenon of anti-Stokes emission of higher energy than energy of absorbed photons is known as a frequency up-conversion. Most often it is observed as infrared-to-visible conversion. The first reports devoted to the anti-Stokes luminescence have appeared in the early 60 's and first materials with such properties were compounds on the base of fluorides $\left(\mathrm{LaF}_{3}, \mathrm{SrF}_{2}, \mathrm{CaF}_{2}, \mathrm{BaF}_{2}\right)$ activated by $\mathrm{Pr}^{3+}$ 
and $\mathrm{Er}^{3+}$ ions [2]. Recently this effect has become extremely interesting because of up-conversion lasers [3].

In the present paper the utilization of up-conversion phenomenon in $\mathrm{Er}-\mathrm{Yb}$ codoped systems for paper security is demonstrated. Laser diodes emitting in the 960-1010 nm range were applied for achievement of a quick visual identification of securities. As active up-convertor hosts, different powdered phosphors can be used (the best not chemically active glasses or fluorides) codoped with $\mathrm{Er}^{3+}$ and $\mathrm{Yb}^{3+}$ ions, for instance YOF or $\mathrm{Li}(\mathrm{K}) \mathrm{YF}_{4}$. The spectral characteristics of active systems are presented and examples of applications are given.

\section{Anti-Stokes emission in the Er-Yb systems}

The phenomenon of up-conversion in the systems of rare earth (RE) ions resulting in occurrence of anti-Stokes emission is well known [1]. It was observed in many crystals and glasses. The most efficient processes were observed in the systems involving $\mathrm{Yb}-\mathrm{Er}, \mathrm{Yb}-\mathrm{Pr}, \mathrm{Yb}-\mathrm{Il}$ ions with $\mathrm{Yb}$ as a sensitizer. In this paper we report the utilization of up-conversion in the powder system of $\mathrm{NaYF}_{4}$ codoped with $\mathrm{Er}$ and $\mathrm{Yb}$ ions. The energy levels of these two ions together with the electronic transitions resulting from cooperative interactions are illustrated in Fig. 1. One can note that after illumination in infrared about $1 \mu \mathrm{m}$ of both

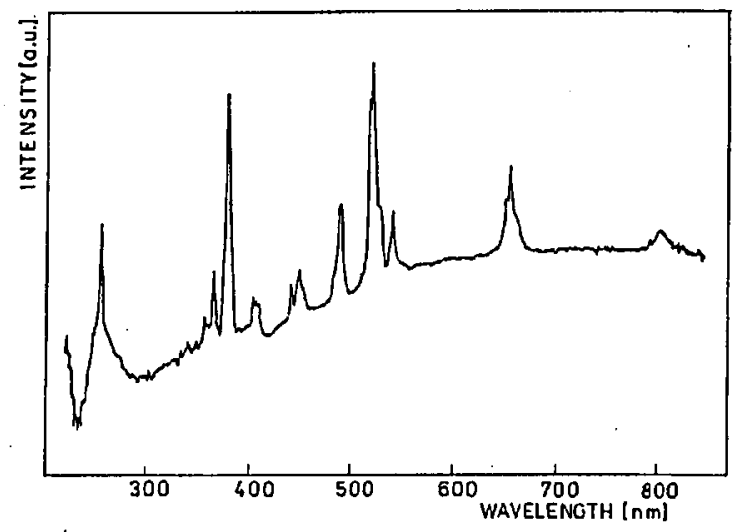

Fig. 1. Schematic energy levels diagrams of $\mathrm{Er}^{3+}$ and $\mathrm{Yb}^{3+}$ ions with energy transitions resulting from cooperative interaction.

neighbours $\mathrm{Yb}^{3+}$ and $\mathrm{Er}^{3+}$ ions the energy from the excited $\mathrm{Yb}^{3+}$ state is transferred cooperatively with the energy from the excited state of the $\mathrm{Er}^{3+}$ ion to the ${ }^{4} F_{7 / 2}$ state of the $\mathrm{Er}^{3+}$ ion and then after relaxation to the lower emitting metastable state ${ }^{4} S_{3 / 2}$ is radiated in visible range.

The reflectance absorption spectrum of the powdered $\mathrm{NaYF}_{4}: \mathrm{Er}, \mathrm{Yb}$ system is presented in Fig. 2.

The anti-Stokes emission spectrum of the powdered $\mathrm{NaYF}_{4}$ : $\mathrm{Er}, \mathrm{YB}$ system pumped by a IR laser diode is shown in Fig. 3. One can note that most intensive emission occurs in the green region. Besides a smaller emission band in the red region is also observed. Both can be utilized for detection of securities. 


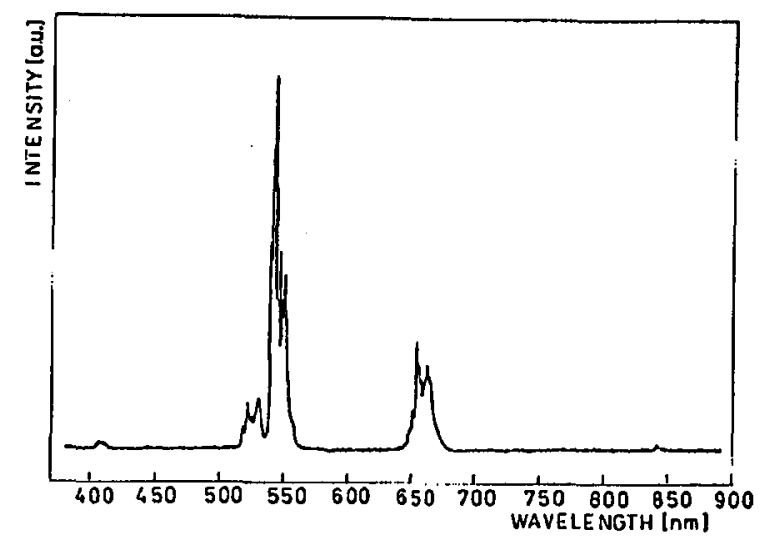

Fig. 2. Reflectance absorption spectrum of powdered $\mathrm{NaYF}_{4}: \mathrm{Er}, \mathrm{Yb}$ system.

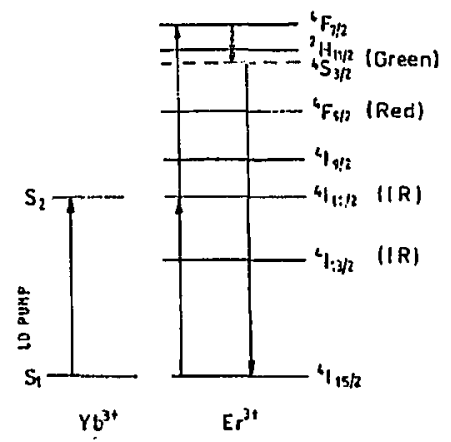

Fig. 3. Emission spectrum of $\mathrm{NaYF}_{4}$ : Er, Yb system under IR laser excitation at $980 \mathrm{~nm}$.

\section{Method of application}

Anti-Stokes emission of Er-Yb doped systems may be used for protection security papers and documents. There are three methods of printing security papers with Er-Yr based components - letterpress, intaglio and offset. Intaglio and letterpress methods are preferred because of possibility to use a relatively large particle size of luminescent pigments $(5 \ldots 70 \mu \mathrm{m})$. The low cost offset method requires less than $5 \mu \mathrm{m}$ of pigment particle size. Unfortunately the decrease in the pigment particle dimensions results in essential reduction of their luminescent efficiency. This imposes on light sources, used for visualization of means of protection, stringent requirements on power as well as spectral and temporal characteristics. The laser diodes are the most attractive light sources for such purpose because of high power, a narrow emission line in the wavelength range of the pigment absorption bands, and simplicity of applying the operation pulse regime. Another features of the laser diodes are low costs and small sizes. 


\section{Device construction}

For a quick visual identification of securities papers we worked out two constructions of the device: desk-top type for office applications and portable pocket device. The desk-top model includes the electronic unit and laser head connected by a flexible twisted cable. The electronic unit consists of the laser driver and analog digital convector (ADC) block. The laser driver bases on a optical feedback circuit with laser diode (LD) built-in photodiode (back facet monitor) providing a reliable $\mathrm{CW}$ operation regime of a $\mathrm{LD}$ and acceptable output power level stability which does not depend on electrical system voltage stability and ambient temperature. Additional steps are also foreseen for the raising immunity interference and circuit protection of the device. The laser power is tuned from zero to maximum rating (up to $100 \mathrm{~mW}$ ) by two sensor keys at the front panel. The LD output power is registered by built-in feedback photodiode. The ADC block converts photocurrent and output power is displayed on the LED monitor at the front panel.

Besides the output power tuning keys and LED monitor the on/off switch, LED on/off indicator and LED indicator of laser diode operation are placed at the front panel.

Additionally the device model with pulse LD operation was constructed for special application. The electronic unit provides adjustable pulse duration from $5 \mathrm{~ns}$ to $100 \mu \mathrm{s}$ with up to $10 \mathrm{kIIz}$ frequency repetition.

The LD head has the laser pointer design. The laser is heat-sunk to the case. The LD on/off button and the LED indicator of laser emission are located on the head case. The optical unit produces the laser beam having stripe shape $(1 \times 5 \mathrm{~mm}$ at $50 \mathrm{~mm}$ from lens). A special unit with optical filter mounted on the laser head allows us to observe the visible luminescence of the tested sample and provides LD infrared irradiation safety.

A pocket device model has the laser pointer design like a laser head of the desk-top device. Two AA type batteries supply the device exploration during 4 hours. Besides the on/off button and the laser emission LED indicator the discharge battery control indicator is located on the pointer case.

\section{Summary}

In this paper we have presented the possibility of utilization of anti-Stokes emission occurring in the system of rare earth ions. As example there were presented the absorption and emission spectra of $\mathrm{NaYF}_{4}$ codoped with $\mathbf{E r}^{3+}$ and $\mathrm{Yb}^{3+}$ ions. This system shows a very efficient green emission after irradiation with infrared laser diode on $0.98 \mathrm{~nm}$. A special construction of IR laser diode device for this purpose is described.

\section{References}

[1] For references on the subject see Spectroscopy of Solids Containing Rare Earth Ions, Eds. A:A. Kaplyanskii, R. Macfarlane, North-Ilolland, New York 1987.

[2] M.R. Brown, W.A. Shand, Phys. Rev. Lett. 11, 366 (1963); ibid. 12, 367 (1964).

[3] A. Silversmith, W. Lenth, R.M. Macfarlane, Appl. Phys. Lett. 45, 1977 (1987); J.Y. Allain, M. Monerie, H. Poignant, Electron. Lett. 26, 166 (1990). 